\title{
Applying Complex Langevin to Lattice QCD at finite
} $\mu$.

\section{K. Sinclair* ${ }^{\dagger}$}

HEP Division, Argonne National Laboratory, 9700 South Cass Avenue, Argonne, Illinois 60439, USA

E-mail: dks@anl.gov

\section{J. B. Kogut}

Department of Energy, Division of High Energy Physics, Washington, DC 20585, USA

and

Department of Physics - TQHN, University of Maryland, 82 Regents Drive, College Park, MD 20742, USA

E-mail: jbkoguteumd.edu

We continue our simulations of lattice QCD at finite quark-number chemical potential, $\mu$, using the complex-Langevin equation (CLE) with gauge-cooling and adaptive updating. The CLE is used because QCD at finite finite $\mu$ has a complex fermion determinant, which prevents use of standard simulation methods. Simulations using the standard lattice action show a transition from hadronic to nuclear matter for $\mu<m_{\pi} / 2$ rather than the expected $\mu \approx m_{N} / 3$. This suggests that the CLE is being influenced by the phase-quenched theory, which has a transition at $\mu=m_{\pi} / 2$. We are therefore performing CLE simulations with a new action which includes an irrelevant chiral 4-fermion interaction. This separates the physics at energies of order of the pion mass and smaller from that at energies of the other hadrons. In doing this, it breaks the extended symmetry of the phase-quenched theory over that of the full theory, raising the masses of the extra pion-like excitations consisting of a quark and a conjugate quark, which could otherwise produce such an anomalous transition. Our preliminary CLE simulations using massless quarks, so that $m_{\pi}=0$, show no transition at $\mu=m_{\pi} / 2=0$, but do show a transition at an appreciably higher value of $\mu$. It remains to be seen if this transition is near to $m_{N} / 3$.

37th International Symposium on Lattice Field Theory - Lattice2019

16-22 June 2019

Wuhan, China

\section{*Speaker.}

${ }^{\dagger}$ This research was supported in part by US Department of Energy contract DE-AC02-06CH11357 


\section{Introduction}

QCD at finite quark-/baryon-number describes nuclear matter. Because QCD at finite quarknumber density has a sign problem, standard methods of simulating it on the lattice, which are based on importance sampling, fail. When one implements finite density by using a quark-number chemical potential, $\mu$, the sign problem manifests itself as a complex fermion determinant. One promising simulation method which can cope with complex 'probabilities' is the complex-Langevin equation (CLE) $[1,2,3,4]$ However, the validity of this method can only be proved if the drift term in the CLE is holomorphic in the fields, and the domain over which the fields vary is exponentially bounded. For (lattice) QCD at finite $\mu$, zeros of the fermion determinant give poles in the drift term, so the drift term is meromorphic, not holomorphic [5, 6, 7, 8, 9, 10, 11, 12, 13]. Hence careful testing of the CLE is needed to determine over what if any range of parameters, the CLE produces correct results. The CLE has been used to study lattice QCD at finite $\mu$ in the heavy-dense limit $[14,15,16,17,18,19]$. Less extensive studies have been made of full lattice QCD at finite $\mu$ $[20,16,21,22,23,24,25,26,27]$.

We have performed CLE simulations of 2-flavour lattice QCD at $\beta=6 / g^{2}=5.6,5.7$ [28] and some preliminary simulations at $\beta=5.8$. Although these indicate that, for sufficiently weak coupling, correct results are obtained for $\mu<<m_{\pi} / 2$ and for $\mu$ large enough to produce saturation, these simulations produce incorrect results for intermediate $\mu$ values. In particular, they show a transition from hadronic to nuclear matter at $\mu<m_{\pi} / 2$ instead of the predicted $\mu \approx m_{N} / 3$. While it is possible that the correct physics might be obtained for sufficiently weak coupling, we are testing modifying the lattice action as a way of producing more physical results.

The action we choose is the standard unimproved staggered-quark action with an additional chiral 4-fermion interaction which preserves the $U(1)$ chiral symmetry of the original action. Since this additional term is an irrelevant operator it should not change continuum physics. This term separates the low energy physics associated with the pion mass from the high energy physics associated with the other hadrons. QCD can then be described in terms of pions, heavy 'constituent' quarks and gluons rather than in terms of light 'current' quarks and gluons. With this action, the additional chiral symmetry breaking of the phase-quenched theory is broken, driving the extra pion-like excitations of a quark and a conjugate quark to higher mass. Our preliminary CLE simulations with this action and massless quarks at finite $\mu$ show no transition at $\mu=m_{\pi} / 2=0$, but do show a transition at a considerably higher $\mu$. It will require simulations of the phase-quenched theory based on this action to decide whether this improved behaviour indicates that this is the expected transition from hadronic to nuclear matter, or whether it is a transition associated with the new phase-quenched theory at half the mass of its heavy pion-like excitations with quark-number 2.

\section{Lattice QCD with a chiral 4-fermion interaction at finite $\mu$}

After introducing auxiliary fields $\sigma$ and $\pi$, our modified Euclidean action for staggered fermions is:

$$
\mathscr{L}=\frac{1}{4} F_{\mu v} F_{\mu v}+\bar{\psi}\left(\not D+\gamma_{4} \mu+\sigma+i \gamma_{5} \tau_{3} \pi+m\right) \psi+\frac{\gamma N_{f}}{8}\left(\sigma^{2}+\pi^{2}\right) .
$$

Such theories have been studied at $\mu=0$ [29], and for $\mu \neq 0[30,31]$. 
After integrating out the fermion fields, the lattice action is

$$
S=\beta \sum_{\square}\left\{1-\frac{1}{6} \operatorname{Tr}\left[U U U U+(U U U U)^{-1}\right]\right\}-\frac{N_{f}}{4} \operatorname{Tr}\{\ln [M(U, \mu, \sigma, \pi)]\}+\frac{N_{f} \gamma}{8} \sum_{\tilde{s}}\left(\sigma^{2}+\pi^{2}\right)
$$

where $(\tilde{s})$ refers to sites on the dual lattice and the Dirac operator $M$ is

$$
M=D(U, \mu)+\frac{1}{16} \sum_{i}\left[\sigma_{i}+i \varepsilon(n) \pi_{i}\right]+m
$$

where $D(U, \mu)$ is the staggered $\not D$ in the presence of quark-number chemical potential $\mu$ and $i$ runs over the 16 sites on the dual lattice adjacent site $n$ and backward links are represented by $U^{-1}$. We shall henceforth refer to this theory as chiral QCD, $\chi \mathrm{QCD}$.

We note that this lattice action has a $U(1)$ chiral symmetry at $m=0$. The action is invariant under the global chiral transformation

$$
\sigma_{i}+i \pi_{i} \rightarrow e^{i \phi}\left[\sigma_{i}+i \pi_{i}\right]
$$

whence

$$
\sigma_{i}+i \varepsilon(n) \pi_{i} \rightarrow e^{i \phi \varepsilon(n)}\left[\sigma_{i}+i \varepsilon(n) \pi_{i}\right] .
$$

Since the Jacobian of this transformation is 1 , the theory is invariant under this transformation. Hence, when $\langle\sigma\rangle$ is non-zero, $\pi$ is a massless Goldstone boson.

The complex Langevin equations are:

$$
-i\left(\frac{d}{d t} U_{l}\right) U_{l}^{-1}=-i \frac{\delta}{\delta U_{l}} S(U, \sigma, \pi)+\eta_{l}
$$

where $\eta_{l}=\eta_{l}^{\alpha} \lambda^{\alpha}$, and

$$
\begin{aligned}
& \frac{d \sigma_{i}}{d t}=-\frac{\delta}{\delta \sigma_{i}} S(U, \sigma, \pi)+\eta_{i}^{\sigma} \\
& \frac{d \pi_{i}}{d t}=-\frac{\delta}{\delta \pi_{i}} S(U, \sigma, \pi)+\eta_{i}^{\pi}
\end{aligned}
$$

$\eta_{l}^{\alpha}, \eta_{i}^{\sigma}, \eta_{i}^{\pi}$ are independent gaussian random numbers appropriately normalized. We discretize this CLE as we did for the standard action, applying gauge-cooling [32] and adaptive updating.

\section{Simulations of lattice $\chi Q C D$ at zero temperature}

We simulate 2-flavour $\left(N_{f}=2\right) \chi \mathrm{QCD}$ with $\beta=5.6, \gamma=5, m=0$ on a $16^{4}$ lattice at finite $\mu$. At $\mu=0$ and for $\mu$ large enough to produce saturation, this lattice is large enough that the theory is in the confined phase, and at a reasonable approximation to zero temperature. We are currently performing simulations at a selection of intermediate $\mu$ values on a $16^{3} \times 36$ lattice to check that $16^{4}$ is adequate to approximate zero temperature. Note, the addition of the 4-fermion interaction does allow us to simulate at $m=0$ where we know that $m_{\pi}=0 . \quad \beta=6 / g^{2}=5.6$ represents a moderate gauge coupling. While $\gamma=5$ represents a relatively large 4 -fermion coupling 
( $\gamma$ is inversely proportional to the 4-fermion coupling), it is not small enough to produce chiralsymmetry breaking $(\chi \mathrm{SB})$ without the gluon interactions. $\gamma=5$ was chosen since it is small enough to allow us to distinguish $\chi \mathrm{SB}$ at $m=0$ on a $16^{4}$ lattice.

To date, we have performed CLE simulations of $\chi \mathrm{QCD}$ with these parameters for $0 \leq \mu \leq 0.6$, performing runs of $2 \times 10^{6}$ updates per $\mu$, except close to the transition where we performed $3 \times 10^{6}$ updates per $\mu$. We see evidence for a phase(?) transition for $\mu \approx 0.35$. We also performed a short simulation at $\mu=1.5$ where we observe saturation.

First we examine the 2 order parameters which measure the chiral condensate. These are $\langle\bar{\psi} \psi\rangle$ and $\langle\sigma\rangle$. These are not independent, but are related by

$$
\langle\bar{\psi} \psi\rangle=\gamma\langle\sigma\rangle .
$$

Because we are simulating at zero quark mass, the direction of the symmetry breaking in the $(\sigma, \pi)$ or $\left(\bar{\psi} \psi, \bar{\psi} \gamma_{5} \xi_{5} \psi\right)$ plane is arbitrary. In fact, because we use a finite lattice, this direction rotates during the simulation, which is how the fact that there is no spontaneous symmetry breaking on a finite lattice is enforced. We therefore use the replacements

$$
\sigma=\sqrt{[\operatorname{real}(\sigma)]^{2}+[\operatorname{real}(\pi)]^{2}}
$$

and

$$
\bar{\psi} \psi=\sqrt{[\operatorname{real}(\bar{\psi} \psi)]^{2}+\left[\operatorname{real}\left(i \bar{\psi} \gamma_{5} \xi_{5} \psi\right)\right]^{2}},
$$

which approach the actual chiral condensates in the large lattice limit. Note $\sigma, \pi, \bar{\psi} \psi$ and $i \bar{\psi} \gamma_{5} \xi_{5} \psi$ are lattice (but not ensemble) averages. We note that now, because of fluctuations, equation 3.1 is only true in the infinite volume limit. Note that the imaginary parts of $\sigma, \pi, \bar{\psi} \psi$ and $i \bar{\psi} \gamma_{5} \xi_{5} \psi$ are very small and have been neglected.
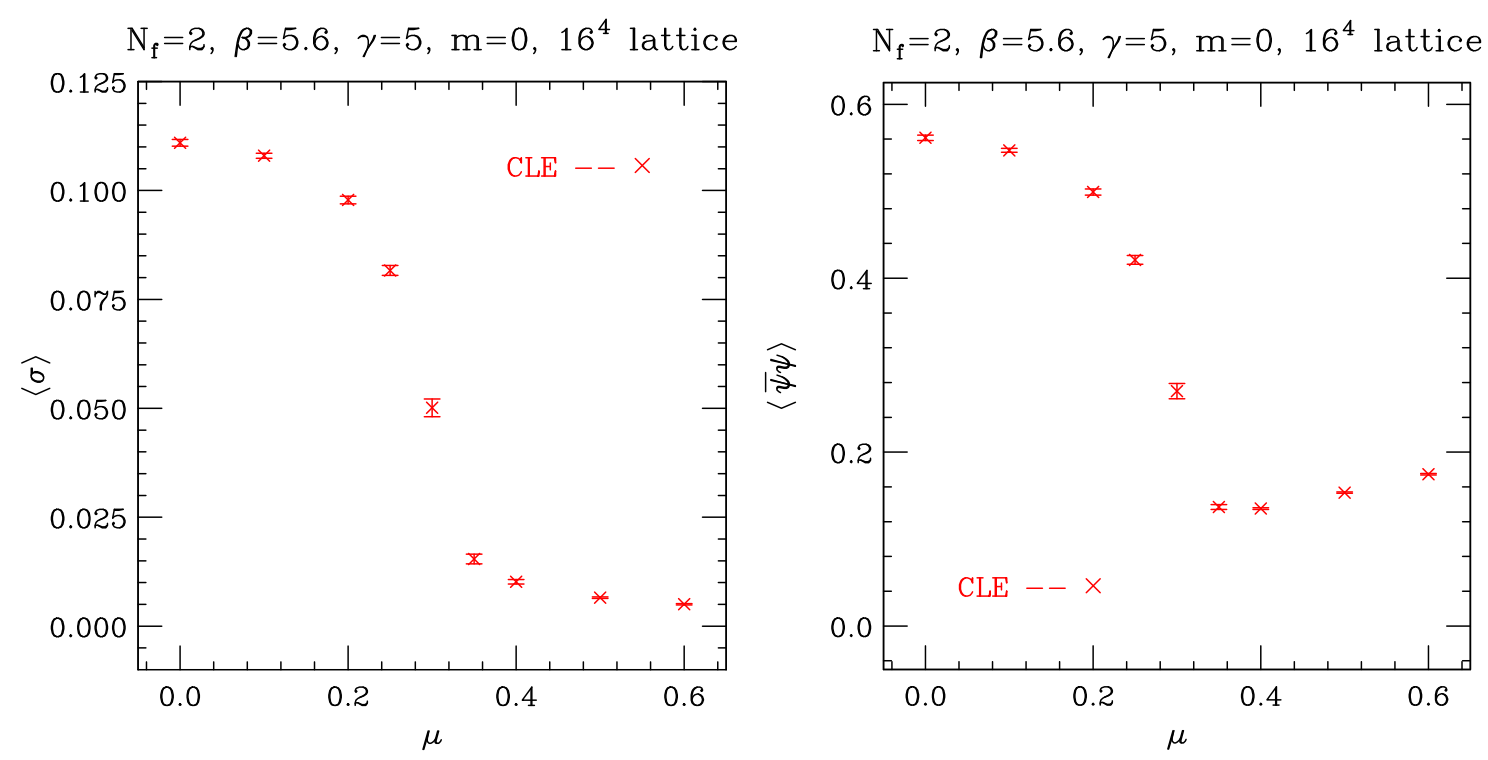

Figure 1: Chiral condensates $\langle\sigma\rangle$ and $\langle\bar{\psi} \psi\rangle$ as functions of $\mu$.

Figure 1 shows the chiral condensates $\langle\sigma\rangle$ and $\langle\bar{\psi} \psi\rangle$ as functions of $\mu$ obtained from our CLE simulations. We observe that there is no sign of a transition at $\mu=m_{\pi} / 2=0$. There is, however, 
a clear sign of a phase transition at $\mu \approx 0.35$. Therefore the addition of the 4-fermion term has removed or moved the transition at an anomalously small $\mu$. This suggests that the transition at $\mu \leq m_{\pi} / 2$, seen in CLE simulations of the original action does indicate that the CLE is being influenced by the phase-quenched theory with its superfluid transition at $\mu=m_{\pi} / 2$. It remains to be seen whether the transition with this new action is the expected hadronic- to nuclear-matter transition driven by nucleons, or the transition of the new phase-quenched theory driven by the condensation of the now-heavy quark-conjugate quark pion-like states of that theory. To test this will require simulating the new phase-quenched theory, and performing spectroscopy with the new action and its phase-quenched counterpart. We note that the chiral condensates do not remain constant up to the transition but rather fall smoothly once $\mu>0$. It remains to be seen if this falloff slows at weaker couplings as is the case with the standard action.

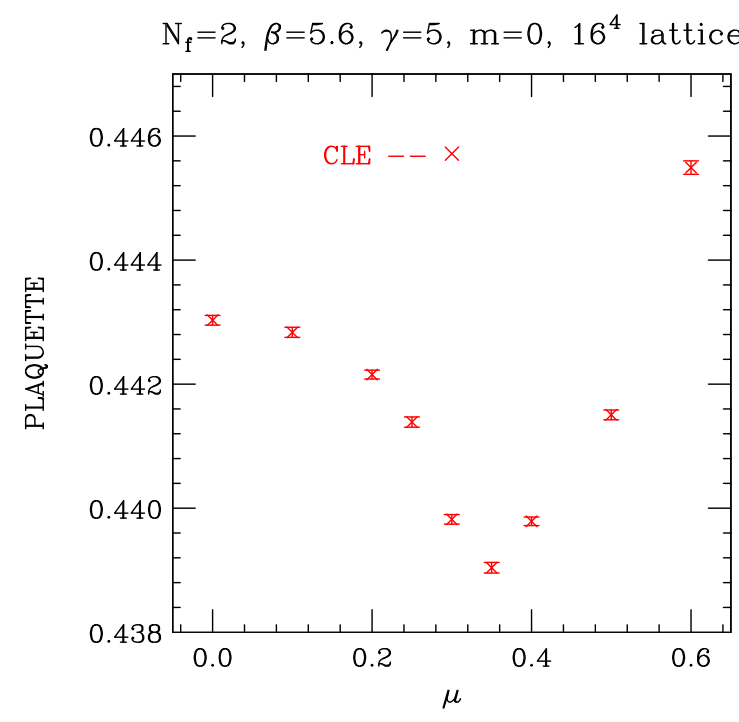

Figure 2: Average plaquette as a function of $\mu$.

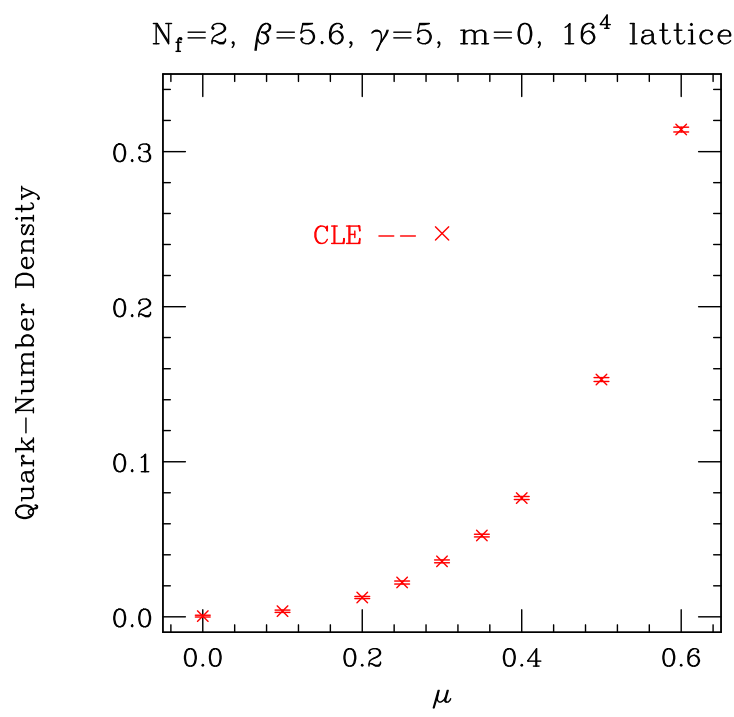

Figure 3: Quark-number density as a function of $\mu$.

Figure 2 shows the average plaquette (Plaquette $=1-\frac{1}{6} \operatorname{Tr}\left[U U U U+(U U U U)^{-1}\right]$ ) as a function of $\mu$. The most striking feature is the sharp minimum at $\mu \approx 0.35$, consistent with the position of the transition in the chiral condensates. Figure 3 shows the quark-number density as a function of $\mu$. It appears to be a smoothly increasing function of $\mu$; the rate of increase increases with $\mu$. For large enough $\mu$ we know that it approaches saturation where the quark-number density is 3 , indicating that all fermion states are filled and the quarks decouple from the gauge fields.

\section{Summary, discussion and conclusions}

Application of the CLE to simulating lattice QCD at finite $\mu$ using the standard staggeredquark lattice QCD action predicts a transition from hadronic to nuclear matter at a $\mu<m_{\pi} / 2$ rather than at the expected $\mu \approx m_{N} / 3$. This suggests that the CLE is influenced by the phasequenched theory with its superfluid transition at $\mu=m_{\pi} / 2$, as is observed in random matrix models $[33,34]$. (Note: there are indications that gauge-cooling might help solve this problem for random matrix models [35].) We are therefore performing CLE simulations using an action incorporating 
a chiral 4-fermion interaction $(\chi \mathrm{QCD})$, which explicitly breaks the additional chiral symmetry of the phase-quenched theory, forcing the masses $m_{\Pi}$ of the pion-like excitations, which give rise to the superfluid transition, to larger values.

Our CLE simulations of $\chi \mathrm{QCD}$ with $m=0$ so that $m_{\pi}=0$ show no transition at $\mu=m_{\pi} / 2=0$, but show strong evidence for a phase transition at a higher $\mu$ value. It remains to be seen if this transition is at $\mu \approx m_{N} / 3$, or at the new superfluid transition of the new phase-quenched theory at $\mu=m_{\Pi} / 2$. This will require simulations of phase-quenched $\chi \mathrm{QCD}$, and spectrum calculations for $\chi \mathrm{QCD}$ and phase-quenched $\chi \mathrm{QCD}$ at $\mu=0$.

We will need to simulate at weaker gauge and 4-fermion coupling to see if this improvement will survive to the continuum limit. In addition we will need to look for evidence that the $\mu$ dependence of observables below the transition weakens with decreasing coupling. Eventually we will need to provide 4-fermion couplings with the full $S U(2) \times S U(2)$ chiral symmetry.

Other attempts to remedy the problems of applying the CLE to simulations of lattice QCD at finite $\mu$ involve either adding additional relevant operators [36] to the QCD action which improve the behaviour of the CLE and taking the limit as these extra operators vanish, or modifying the dynamics by adding irrelevant terms to the drift term [37]. Since these irrelevant terms have no domain of holomorphicity, one must take the limit as these extra terms vanish.

\section{References}

[1] G. Parisi, Phys. Lett. B 131, 393 (1983).

[2] J. R. Klauder, Acta Phys. Austriaca Suppl. 25, 251 (1983).

[3] J. R. Klauder, J. Phys. A 16, L317 (1983).

[4] J. R. Klauder, Phys. Rev. A 29, 2036 (1984).

[5] G. Aarts, E. Seiler and I. O. Stamatescu, Phys. Rev. D 81, 054508 (2010)

[6] G. Aarts, F. A. James, E. Seiler and I. O. Stamatescu, Eur. Phys. J. C 71, 1756 (2011)

[7] K. Nagata, J. Nishimura and S. Shimasaki, PTEP 2016, no. 1, 013 B01 (2016)

[8] J. Nishimura and S. Shimasaki, Phys. Rev. D 92, no. 1, 011501 (2015)

[9] K. Nagata, J. Nishimura and S. Shimasaki, Phys. Rev. D 94, no. 11, 114515 (2016)

[10] G. Aarts, E. Seiler, D. Sexty and I. O. Stamatescu, JHEP 1705, 044 (2017)

[11] E. Seiler, EPJ Web Conf. 175, 01019 (2018)

[12] G. Aarts, K. Boguslavski, M. Scherzer, E. Seiler, D. Sexty and I. O. Stamatescu, EPJ Web Conf. 175, 14007 (2018)

[13] K. Nagata, J. Nishimura and S. Shimasaki, JHEP 1805, 004 (2018)

[14] G. Aarts and I. O. Stamatescu, JHEP 0809, 018 (2008)

[15] G. Aarts, L. Bongiovanni, E. Seiler, D. Sexty and I. O. Stamatescu, Eur. Phys. J. A 49, 89 (2013)

[16] G. Aarts, E. Seiler, D. Sexty and I. O. Stamatescu, Phys. Rev. D 90, no. 11, 114505 (2014)

[17] G. Aarts, F. Attanasio, B. Jäger and D. Sexty, JHEP 1609, 087 (2016)

[18] J. Langelage, M. Neuman and O. Philipsen, JHEP 1409, 131 (2014) 
[19] T. Rindlisbacher and P. de Forcrand, JHEP 1602, 051 (2016)

[20] D. Sexty, Phys. Lett. B 729, 108 (2014)

[21] Z. Fodor, S. D. Katz, D. Sexty and C. Török, Phys. Rev. D 92, no. 9, 094516 (2015)

[22] K. Nagata, H. Matsufuru, J. Nishimura and S. Shimasaki, PoS LATTICE 2016, 067 (2016)

[23] S. Tsutsui, Y. Ito, H. Matsufuru, J. Nishimura, S. Shimasaki and A. Tsuchiya, PoS LATTICE 2018, $144(2018)$

[24] M. Scherzer, E. Seiler, D. Sexty and I. O. Stamatescu, PoS LATTICE 2018, 320 (2019)

[25] Y. Ito, H. Matsufuru, J. Nishimura, S. Shimasaki, A. Tsuchiya and S. Tsutsui, PoS LATTICE 2018, 146 (2018)

[26] S. Tsutsui, Talk presented by S. Tsutsui at Lattice 2019, Wuhan (2019)

[27] D. Sexty, Phys. Rev. D 100, no. 7, 074503 (2019)

[28] J. B. Kogut and D. K. Sinclair, Phys. Rev. D 100, no. 5, 054512 (2019)

[29] J. B. Kogut, J. F. Lagae and D. K. Sinclair, Phys. Rev. D 58, 034504 (1998)

[30] I. M. Barbour, J. B. Kogut and S. E. Morrison, Nucl. Phys. Proc. Suppl. 63, 436 (1998)

[31] M. Chavel, Phys. Rev. D 56, 5596 (1997)

[32] E. Seiler, D. Sexty and I. O. Stamatescu, Phys. Lett. B 723, 213 (2013)

[33] A. Mollgaard and K. Splittorff, Phys. Rev. D 88, no. 11, 116007 (2013)

[34] J. Bloch, J. Glesaaen, J. J. M. Verbaarschot and S. Zafeiropoulos, JHEP 1803, 015 (2018)

[35] K. Nagata, J. Nishimura and S. Shimasaki, JHEP 1607, 073 (2016)

[36] K. Nagata, J. Nishimura and S. Shimasaki, Phys. Rev. D 98, no. 11, 114513 (2018)

[37] F. Attanasio and B. Jäger, Eur. Phys. J. C 79, no. 1, 16 (2019) 